\title{
Hyperparabolic concentrators
}

\author{
Angel García-Botella, ${ }^{1, *}$ Antonio Álvarez Fernández-Balbuena, ${ }^{2}$ Daniel Vázquez, ${ }^{2}$ \\ Eusebio Bernabeu, ${ }^{3}$ and Agustín González-Cano ${ }^{2}$ \\ ${ }^{1}$ Departamento Física Aplicada a los Recursos Naturales, Universidad Politécnica de Madrid, \\ Escuela Técnica Superior de Ingenieros de Montes, Ciudad Universitaria s/n, \\ 28040 Madrid, Spain \\ ${ }^{2}$ Departamento de Óptica, Universidad Complutense de Madrid, Escuela Universitaria Óptica, \\ Arcos de Jalón, s/n, 28037 Madrid, Spain \\ ${ }^{3}$ Departamento de Óptica, Universidad Complutense de Madrid, Facultad Ciencias Físicas, \\ Ciudad Universitaria s/n, 28040 Madrid, Spain \\ ${ }^{*}$ Corresponding author: angel.garciab@upm.es
}

Received 7 November 2008; accepted 10 December 2008;

posted 11 December 2008 (Doc. ID 103833); published 23 January 2009

\begin{abstract}
We present a family of three-dimensional concentrators constructed from the photic field generated by a Lambertian emitter. The profile of these concentrators is obtained from the field lines for a two-dimensional truncated wedge and is based on the union between a hyperbola and a tilted parabola. By revolution of this profile, we obtain hyperparabolic concentrators (HPCs). In the limiting case when the focal length of the hyperbola becomes the radius of the exit aperture, the HPC becomes the well-known compound parabolic concentrator. On the other hand, when the focal length of the hyperbola becomes infinite, the HPC achieves the thermodynamic limit of concentration. (C) 2009 Optical Society of America

OCIS codes: $\quad 220.1770,350.6050,350.4600$.
\end{abstract}

\section{Introduction}

Optical concentrators, illuminators, and couplers have actual and potential applications and much effort has been made to improve the behavior of these components [1,2]. Nonimaging optics is a field devoted to the design of such components, where light collecting systems are used instead of the usual imaging systems. One of the most powerful design techniques for nonimaging optical devices is the so-called flow-line method. Winston and Welford introduced the concept of the geometric vector flux $\mathbf{J}$ [3], where the direction of $\mathbf{J}$ is the flow line, and showed that ideal flux concentrators have shapes that do not disturb the geometric vector flux field. On the other hand, Moon and Spencer [4] used an analogous concept, the pharosage vector, to study the vector flux field from Lambertian illuminators, developing the

0003-6935/09/040712-04\$15.00/0

(C) 2009 Optical Society of America so-called photic field theory, that has been used to design concentrators $[5,6]$. The analogy between these two methods is due to the fact that flux and field are intimately related concepts. One important capability of this field approach is that it can provide higherorder solutions; in our case, higher-order nonimaging optics components.

The flow-line method has been used to study the three-dimensional (3D) compound parabolic concentrator (CPC) [7], as a component originating from the field of a Lambertian emitter in the form of a $2 \mathrm{D}$ truncated wedge. After that, many developments and modifications of the CPC were studied [1], including a dielectric-filled CPC, a truncated CPC, and a two-stage CPC. Shatz and Bortz studied a global optimization procedure [1] to obtain reflective concentrators having superior performance to that of the 3D CPC. We focus our interest on the study of the field from a Lambertian emitter in the form of a two-dimensional (2D) truncated wedge, and we show that the flow lines of this field define a 
new higher-order family of concentrators, the HPCs, which improves on the performance of the 3D CPC, which is the lower limit of performance of the 3D HPC, and having the thermodynamic limit of concentration as the theoretical upper limit of performance.

In Section 2 we present the geometric design of the concentrators based on the flow-line design method. In Section 3 we analytically characterize the HPC. In Section $\overline{4}$ we study the behavior of the 3D HPC by ray-tracing simulations, showing the thermodynamic limit as the upper limit of performance. Finally, conclusions are given in Section 5 .

\section{Hyperparabolic Concentrator}

The flow-line method was introduced by Winston and Welford [3] and studied by several authors [7,8]. It concludes that a way to design a concentrator with maximum theoretical concentration is to place mirrors in the flow lines of a specific Lambertian emitter. A classical application was to evaluate the flow lines for a $2 \mathrm{D}$ truncated wedge [7], from which the CPC geometry was obtained, as the flow line that starts at the vertex of the wedge (focus of the tilted parabola). Considering this photic field, we can study the flow lines that start at the upper part of the wedge, not at the vertex; see Fig. 1. These flow lines produce new concentrators, which are HPCs, with the same acceptance angle as a CPC but with a smaller exit aperture diameter. These new concentrators have the shape of a hyperbola continuously joined with a tilted parabola, with its focus at the vertex of the wedge. Figure $\underline{1}$ shows that the profile has an in-

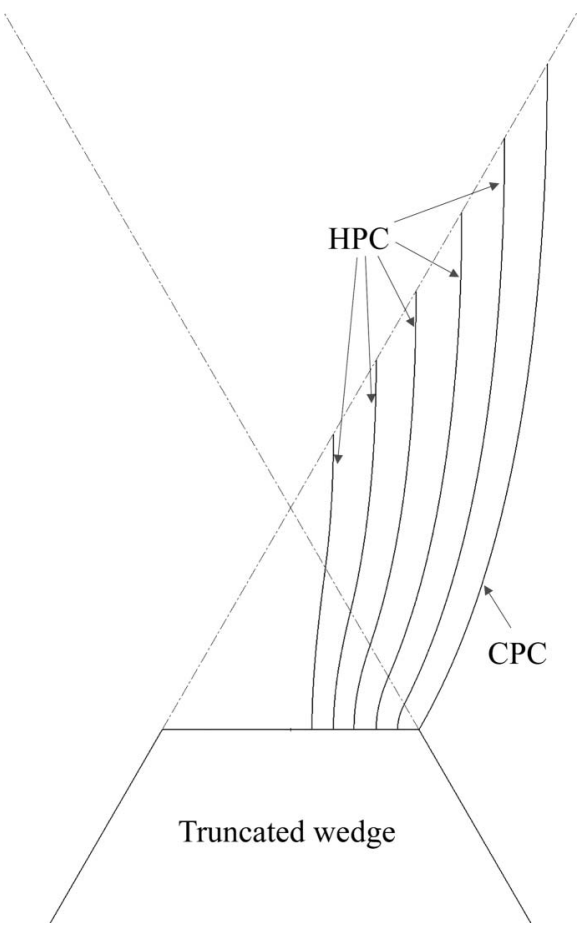

Fig. 1. Flow lines for the 2D truncated wedge, it shows HPC and CPC profiles. flection point, which proves that its order is higher than 2 . The union between these two conics must fulfill three conditions: first, the focus of the hyperbola, $F^{\prime}$, and the focus of the parabola, $F_{p}$, must coincide; second, the union point $U$ is the intersection point between the hyperbola and a line that passes through the nearest focus of the hyperbola, $F$, and cuts the axis of the hyperbola at an angle $\theta$; and third, the axis of the parabola, which passes through its focus $F_{p}$, cuts the axis of the hyperbola at an angle $\theta$. These conditions ensure a continuous union between both conics. The starting and the ending points of the profile have 0 slope; Fig. 2 shows a sketch of this profile. With this geometry, in the limiting case when the focal length of the hyperbola is the radius of the exit aperture, $f=a^{\prime}$, the HPC becomes the CPC. By its geometric design, it is easy to prove that the $2 \mathrm{D} \mathrm{HPC}$ is an ideal concentrator. All the rays incident on the entry aperture of a 2D HPC at angles minor or equal to $\theta$ will be redirected, by the parabolic section of the concentrator, to the segment $F-F^{\prime}$, and their second and successive reflections will cause them to emerge from the concentrator.

The construction of HPCs can be done by rotational symmetry (3D HPC), by translational symmetry (2D HPC), or by elliptical symmetry [9] (elliptical HPC). Figure 3 shows three 3D HPCs with different values for the hyperbola focal length $f$ and the same value for acceptance angle $\theta$ and exit aperture radius $a^{\prime}$.

\section{Analytical Characterization of the Hyperparabolic Concentrator}

The HPC must be geometrically defined by three parameters, unlike the CPC, which needs only two parameters to be defined. The parameters of the HPC are $f$, the focal length of the hyperbola, $\theta$, the acceptance angle, and $\alpha^{\prime}$, the radius of the exit aperture. These parameters completely determine the shape and the overall length of the HPC as functions of its parameters (Fig. 2):

$$
L=\left(f+\frac{a^{\prime}}{\sin \theta}\right) \cot \theta
$$

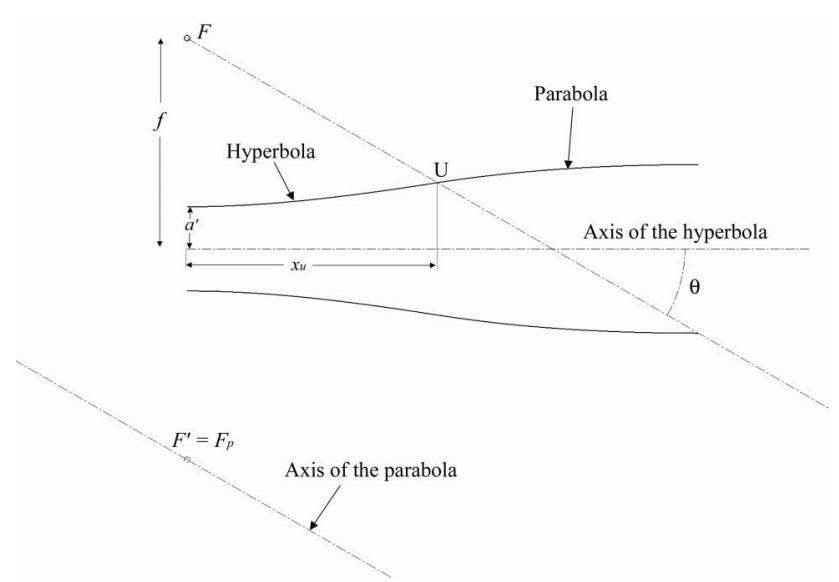

Fig. 2. Geometric profile of the HPC. 


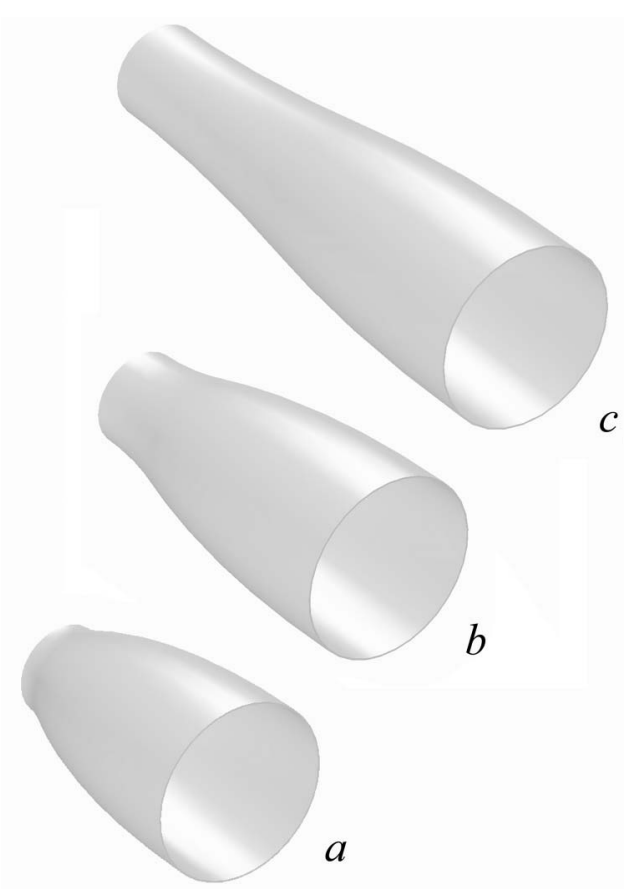

Fig. 3. Three 3D HPCs with different hyperbola focal lengths of $f_{a}=18 \mathrm{~mm}, f_{b}=30 \mathrm{~mm}$, and $f_{c}=60 \mathrm{~mm}$, and the same acceptance angle $\theta=30^{\circ}$ and radius of exit aperture $a^{\prime}=12 \mathrm{~mm}$.

The union point between the hyperbola and the parabola, which defines the length of the hyperbolic part of the concentrator $x_{u}$ (Fig. 2), can be calculated by the intersection between the hyperbola and the line that passes through the focus. Using the HPC parameters, it is

$$
x_{u}=\frac{\left(f^{2}-a^{\prime 2}\right)\left(f \tan \theta-a^{\prime} \sec \theta\right)}{\left(f^{2} \tan ^{2} \theta-a^{\prime 2} \sec ^{2} \theta\right)} .
$$

This point is the inflection point in the profile of the concentrator. By this construction, the parabola has a focal length of

$$
f_{p}=\left(f+\frac{a^{\prime}}{\sin \theta}\right) \sin \theta=f \sin \theta+a^{\prime}
$$

It is also possible to write the equation of the HPC as an implicit function of its parameters, the hyperbolic part and tilted parabolic part,

$$
\begin{aligned}
\frac{y^{2}}{a^{2}}- & \frac{x^{2}}{f^{2}-a^{2}}=1, \quad 0 \leq x \leq x_{u}, \\
& {[(y+f) \cos \theta+x \sin \theta]^{2}=4\left(f \sin \theta+a^{\prime}\right) } \\
\times & {\left[x \cos \theta-(y+f) \sin \theta+f \sin \theta+a^{\prime}\right], } \\
& x_{u} \leq x \leq L .
\end{aligned}
$$

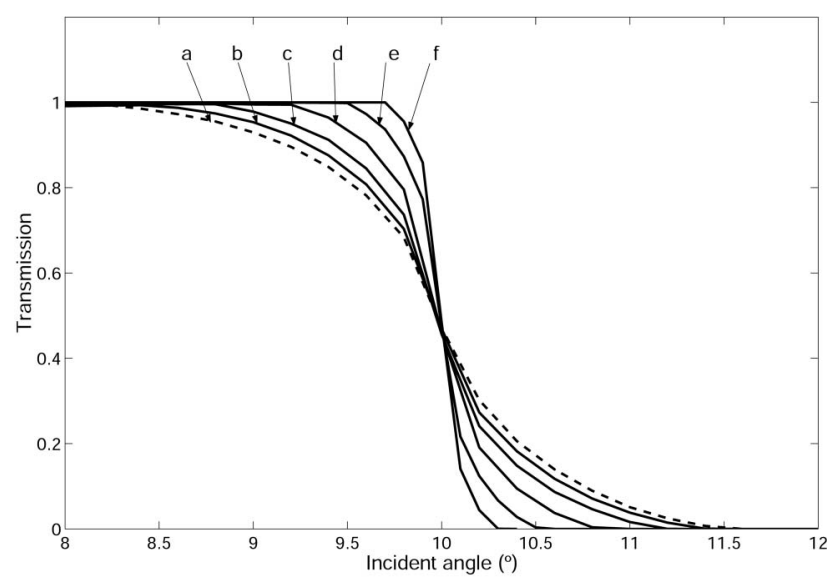

Fig. 4. Transmission-angle curves for six 3D HPCs with $\theta=10^{\circ}$ $a^{\prime}=12 \mathrm{~mm}$ : CPC (a, dashed curve), $f=18 \mathrm{~mm}$ (curve b), $f=$ $30 \mathrm{~mm}$ (curve c), $f=60 \mathrm{~mm}$ (curve d), $f=120 \mathrm{~mm}$ (curve e), and $f=240 \mathrm{~mm}$ (curve $\mathrm{f}$ ).

\section{Transmission-Angle Curves for Hyperparabolic Concentrators}

To study the behavior of the 3D HPC, we computed the transmission properties by ray-tracing simulations [10] for different parametric configurations. We studied the dependence on the transmissionangle curve versus the focal length of the hyperbola for three different acceptance angles, $\theta=10^{\circ}$, $\theta=30^{\circ}$, and $\theta=50^{\circ}$. The dependence on the radius of the exit aperture $a^{\prime}$ is basically a scale factor. The way to build scaled HPCs with the same acceptance angle $\theta$ and the same transmission-angle curve is to multiply, by a scale factor of $N$, not only the $a^{\prime}$ parameter, as in the CPC, but also the $f$ parameter. Figure 4 plots transmission-angle curves for six 3D HPCs with acceptance angle $\theta=10^{\circ}$, radius $a^{\prime}=12 \mathrm{~mm}$, and reflectance $\rho=1$, these curves correspond to a CPC (curve a), a HPC with $f=18 \mathrm{~mm}$ (curve b), a HPC with $f=30 \mathrm{~mm}$ (curve c), a HPC with $f=60 \mathrm{~mm}$ (curve d), a HPC with $f=120 \mathrm{~mm}$ (curve e), and a HPC with $f=240 \mathrm{~mm}$ (curve f). It

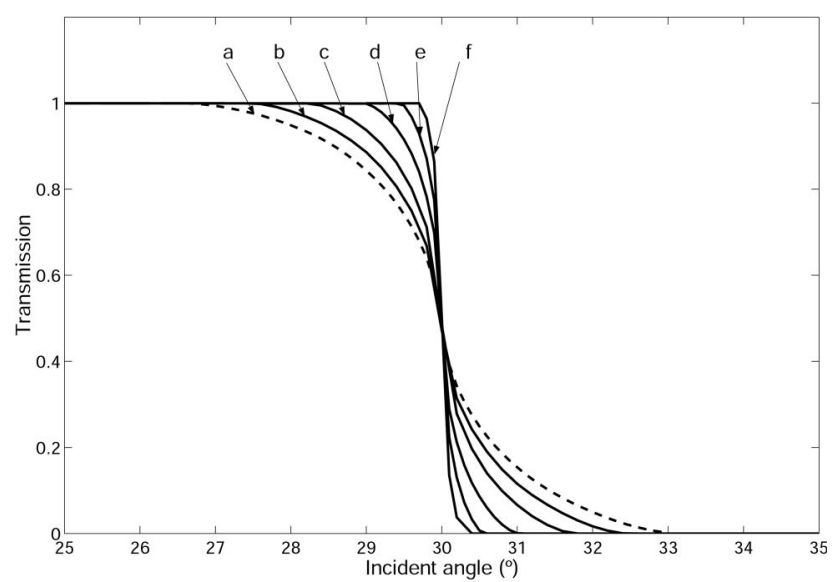

Fig. 5. Transmission-angle curves for six 3D HPCs with $\theta=30^{\circ}$, $a^{\prime}=12 \mathrm{~mm}$ : CPC (a, dashed curve), $f=18 \mathrm{~mm}$ (curve b), $f=$ $30 \mathrm{~mm}$ (curve c), $f=60 \mathrm{~mm}$ (curve d), $f=120 \mathrm{~mm}$ (curve e) and $f=$ $240 \mathrm{~mm}$ (curve f). 


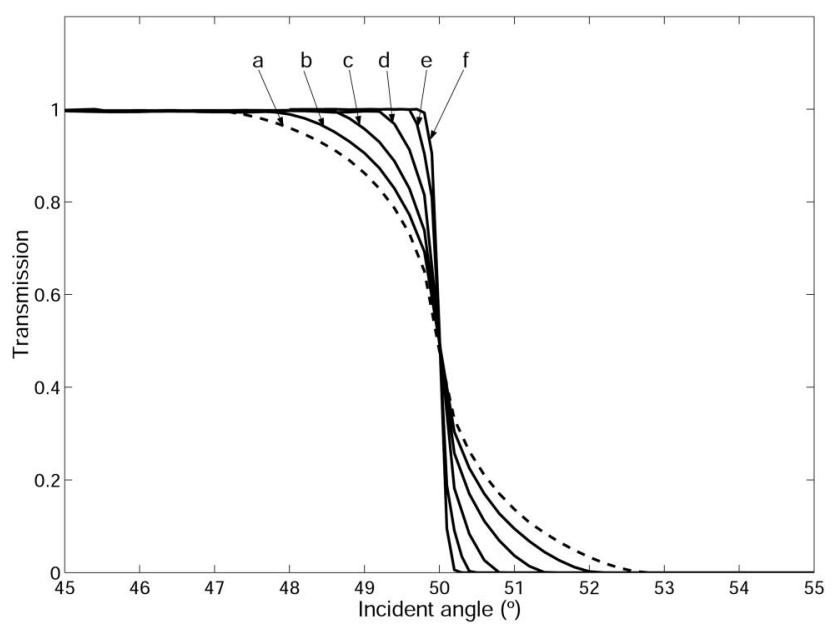

Fig. 6. Transmission-angle curves for six $3 \mathrm{D}$ HPCs with $\theta=50^{\circ}$, $a^{\prime}=12 \mathrm{~mm}$ : CPC (a, dashed curve), $f=18 \mathrm{~mm}$ (curve b), $f=30 \mathrm{~mm}$ (curve c), $f=60 \mathrm{~mm}$ (curve d), $f=120 \mathrm{~mm}$ (curve e) and $f=240 \mathrm{~mm}$ (curve $\mathrm{f}$ ).

shows that the CPC transmission-angle curve becomes the lower limit of performance of the HPCs, and that the total transmission of the concentrators increases with $f$. At the theoretical limit of $f \rightarrow \infty$, the 3D HPC behaves as an ideal infinite source concentrator, with a step transmission-angle curve, thus achieving the thermodynamic limit of concentration in accordance with Garwin's conclusion [11]. Similar transmission-angle curves have been computed for HPCs with acceptance angles of $\theta=30^{\circ}$ (Fig. $\underline{5}$ ) and $\theta=50^{\circ}$ (Fig. 6).

\section{Conclusions}

We have presented a new 3D infinite source family of concentrators, the HPCs. The design is based on the application of the field method to a particular geometry, the truncated wedge. The HPC profile is made up by the continuous union between a hyperbola and a tilted parabola with its foci at the same point, the vertex of the wedge. From the analytical point of view, the HPC is a higher-order concentrator that must be defined by three parameters, focal length of the hyperbola $f$, acceptance angle $\theta$, and radius of the exit aperture $a^{\prime}$. We have studied the transmission-angle curves by ray-tracing simulations for different configurations, showing that the lower limit of performance for a HPC is the CPC and the theoretical upper limit of performance is the thermodynamic limit of concentration.

\section{References}

1. R. Winston, J. C. Miñano, and P. Benitez, with contributions by N. Shatz and J. C. Bortz, Nonimaging Optics (Elsevier Academic, 2005).

2. D. Jenkins, R. Winston, J. Bliss, J. O'Gallagher, A. Lewandowski, and C. Bingham, "Solar concentration of 50000 achieved with output power approaching $1 \mathrm{~kW}$," J. Sol. Energy Eng. 118, 141-145 (1996).

3. R. Winston and W. T. Welford, "Geometrical vector flux and some new nonimaging concentrators," J. Opt. Soc. Am. 69, 532-536 (1979).

4. P. Moon and D. E. Spencer, Photic Field (Massachusetts Institute of Technology Press, 1981).

5. J. O'Gallagher and R. Winston, "Test of a trumpet secondary concentrator with a paraboloidal dish primary," Sol. Energy 36, 37-44 (1986).

6. A. Garcia-Botella, A. A. Fernandez-Balbuean, D. Vázquez, and E. Bernabeu, "Ideal 3D asymmetric concentrator," Sol. Energy 83, 113-117 (2009).

7. R. Winston and W. T. Welford, "Ideal flux concentrators as shapes that do not disturb the geometrical vector flux field: a new derivation of the compound parabolic concentrator," J. Opt. Soc. Am. 69, 536-539 (1979).

8. P. Greenman, "Geometrical vector flux sinks and ideal flux concentrators," J. Opt. Soc. Am. 71, 777-779 (1981).

9. A. Garcia-Botella, A. A. Fernanndez-Balbuena, and E. Bernabeu, "Elliptical concentrators," Appl. Opt. 45, 7622-7627 (2006).

10. Tracepro software, http://www.lambdares.com/.

11. R. L. Garwin, "The design of liquid scintillation cells," Rev. Sci. Instrum. 23, 755-757 (1952). 